\title{
Combined percutaneous approach for aortic valve implantation in poor vascular access
}

\author{
Kala P, Tesar D, Hajek P, Adlova R, Veselka J \\ Department of Cardiology, Cardiovascular Center, 2nd Faculty of Medicine and University Hospital Motol, \\ Charles University, Prague, Czech Republic. petr.kala@fnmotol.cz
}

\section{ABSTRACT}

Transcatheter aortic valve implantation (TAVI) is a well-established management option for symptomatic patients with severe aortic stenosis. The minimally invasive transfemoral approach is considered to be superior to non-transfemoral accesses; however, its use is often limited by concomitant peripheral artery disease (PAD). Percutaneous transluminal angioplasty with stent implantation (PTA) is a gold-standard therapy for symptomatic PAD. We present 2 cases from our cohort of patients with severe aortic stenosis and PAD previously contraindicated for TAVI because of poor peripheral vascular access. However, the patients were eventually treated either by staged PTA and TAVI through an endothelialized stent or PTA and TAVI though a newly implanted peripheral stent during one procedure. We provide recommendations based on our experience of how to select the optimal patients for such a combined minimally invasive transfemoral approach (Fig. 2, Ref. 9). Text in PDF www.elis.sk

KEY WORDS: transcatheter valve implantation, peripheral arterial disease, aortic valve disease, percutaneous intervention, atherosclerosis.

\section{Introduction}

Aortic stenosis is the most common valve disease in Europe and North America that leads to catheter intervention, with a growing prevalence due to the ageing population (1). Transcatheter aortic valve implantation (TAVI) is a well-established management option for symptomatic patients with a severe aortic stenosis at a high surgical risk $(1,2)$. Recent studies showed a shortand mid-term benefit also for intermediate- and low-risk patients $(3,4,5,6)$. Retrograde transfemoral access is the gold-standard because of its safety and the option of a minimally invasive fully percutaneous TAVI with the patient under a conscious sedation or local anaesthesia (7). Other alternative non-transfemoral approaches have been developed and investigated, especially for patients without a suitable peripheral femoral access. Although no randomized controlled trials (RCT) have compared transfemoral and non-transfemoral access routes, observational data showed that 30-day and 1-year mortality, respectively, were nearly twice (4.7 vs $8.1 \%$ ) as high and one and a half (16.4 vs $24.8 \%$ ) times higher in a non-transfemoral approach than in a transfemoral (8).

We identified 6 patients at our centre, who had poor a peripheral vascular access and who underwent either simultaneous peripheral

Department of Cardiology, Cardiovascular Center, 2nd Faculty of Medicine and University Hospital Motol, Charles University, Prague, Czech Republic

Address for correspondence: P. Kala, MD, Cardiovascular Center, University Hospital Motol, V Uvalu 84, CZ-150 06 Prague, Czech Republic. Phone: +420.702024181 angioplasty during a TAVI procedure or earlier before the TAVI procedure as an access-preparation strategy. We present here one case from each group.

\section{Patient 1}

A 69-year-old male patient with a generalized atherosclerosis and many comorbidities was referred to our cardiovascular center with a chronic intermittent claudication and worsening effort dyspnoea due to severe aortic stenosis confirmed by echocardiography. TAVI was suggested as the treatment of choice. However, poor peripheral vascular access was revealed bilaterally. We preceded with percutaneous transluminal angioplasty (PTA) of the left and right external iliac arteries with implantation of two stents (Wallstent 9/40 mm, Boston Scientific, MA, United States; Hippocampus $7 / 15 \mathrm{~mm}$, Medtronic, Minneapolis, MN, United States) (Figure 1). Three months later, the patient successfully underwent TAVI (ACCURATE Neo L, Boston Scientific, MA, United States) through a right femoral access and fully endothelialized peripheral stent (Fig. 1).

\section{Patient 2}

A 77-year-old male patient with a generalized atherosclerosis was admitted to our hospital due to new onset of angina pectoris. Echocardiography revealed a severe aortic stenosis and coronary angiography was stable without any new stenosis. A focal calcification of right iliac artery was described on computed tomography $(\mathrm{CT})$, however according to the evaluation, the diameters 

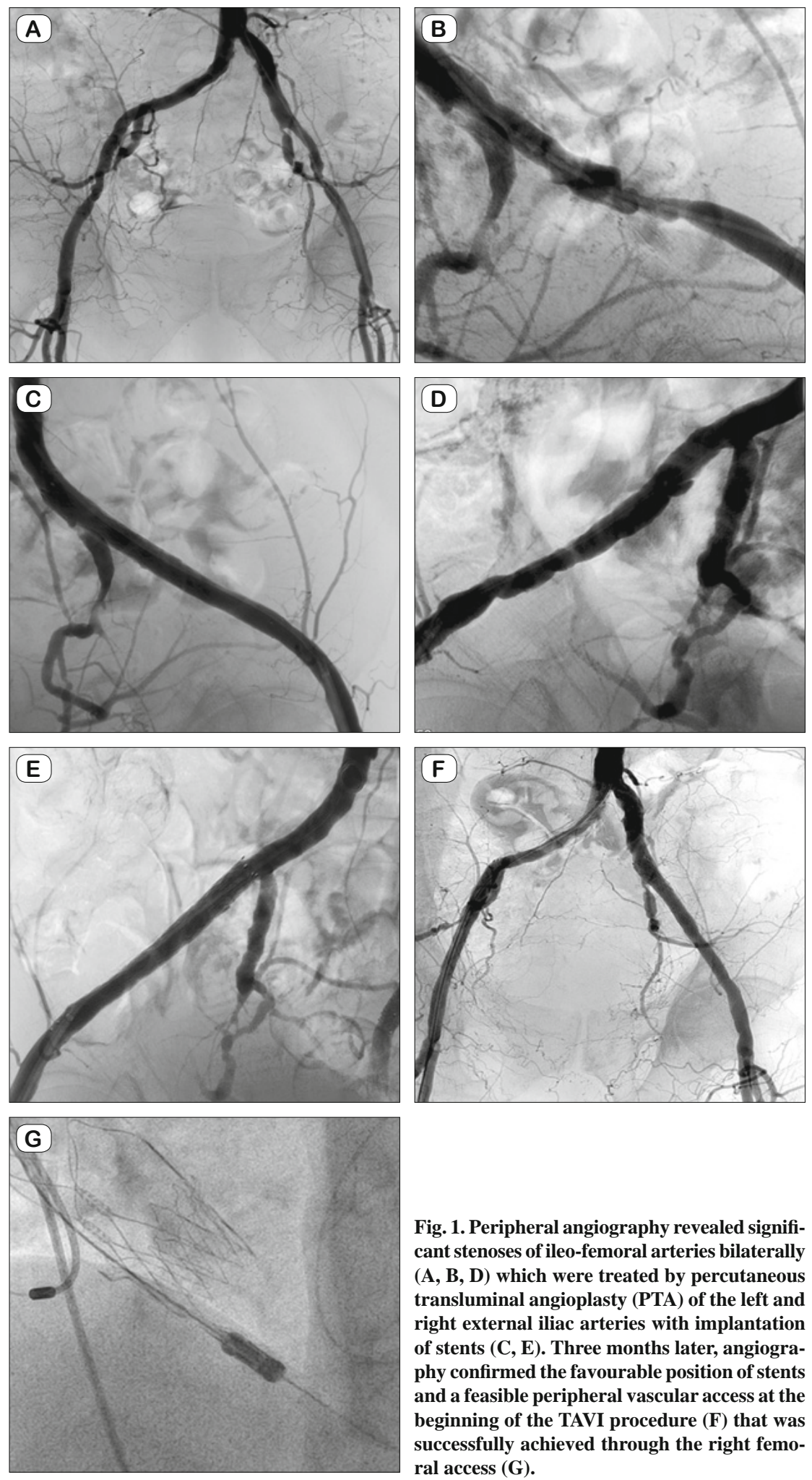

Fig. 1. Peripheral angiography revealed signifcant stenoses of ileo-femoral arteries bilaterally (A, B, D) which were treated by percutaneous transluminal angioplasty (PTA) of the left and right external iliac arteries with implantation of stents (C, E). Three months later, angiography confirmed the favourable position of stents and a feasible peripheral vascular access at the beginning of the TAVI procedure $(F)$ that was successfully achieved through the right femoral access (G).

were sufficient for TAVI therefore transfemoral TAVI was indicated. After the beginning of the procedure, the delivery system was unable to go through the calcified part of the iliac artery, therefore peripheral vascular intervention was selected. At first, balloon dilatation ( $8 \mathrm{~mm}$ and $10 \mathrm{~mm}$ peripheral balloon) was performed and then a peripheral vascular stent was implanted due to a modest dissection of the right common iliac artery. Through the right femoral artery and then the right iliac artery with the implanted stent, the delivery catheter system for the Accurate $M$ valve (Boston Scientific, MA, United States) was introduced, and the valve was implanted at the aortic position (Fig. 2).

\section{Discussion}

We present a unique case report of patients with a poor peripheral vascular access to undergo TAVI performed by using the strategy of preparation of the peripheral arterial side by peripheral angioplasty and then delivery of TAVI either during one procedure or staged after endothelization of stents. We suggest this might be an option for previously contraindicated patients, who had a poor peripheral vascular access or an attempt to avoid alternative approaches (transapical, transaxillar, transcarotidal), which are associated with greater complications and worse outcomes (8). However, there are potential disadvantages, which need to be mentioned. A complex percutaneous procedure might lead to higher consumption of contrast dye and prolongation of fluoroscopy time. The result is uncertain because the intended peripheral angioplasty might not be successful for several reasons (multifocal, calcified lesions, too small or tortuous vessel) and might not create a path for the transcatheter delivery system. Calcifications on CT scan should be evaluated carefully although the measured arterial diameter is sufficient for TAVI and the team should be ready for the potential combined procedure (peripheral angioplasty and TAVI). Therefore, an outstanding preliminary evaluation process and extensive experience with peripheral angioplasty among the "heart team" are necessary.

One or two short focal peripheral stenoses might be treated by stenting or balloon dilatation. Conversely, multifocal or casting calcified lesions are unsuitable for such a strategy. Also, the 

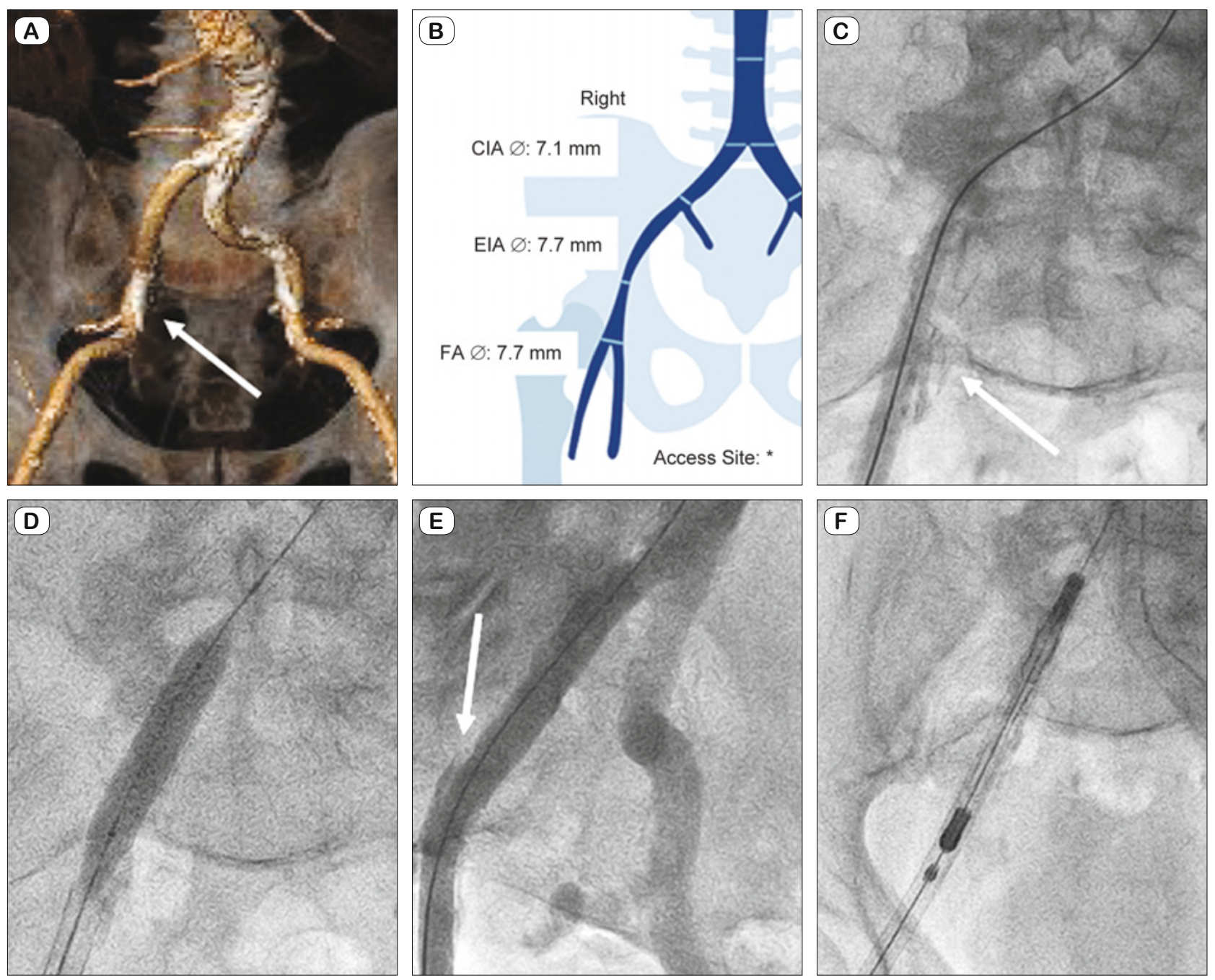

Fig. 2. A focal calcification (arrow A, C) of the right iliac artery was described on computed tomography (CT) scan (A), however, according to CT evaluation, the diameters were sufficient for TAVI (B) therefore TAVI procedure was indicated. After the beginning of the procedure, the delivery system was unable to go through the calcified part (arrow $C$ ) of the iliac artery, therefore peripheral vascular intervention was selected. At first, balloon dilatation ( $8 \mathrm{~mm}$ and $10 \mathrm{~mm}$ peripheral balloon) was performed (D) and then a peripheral vascular stent was implanted due to a modest dissection (arrow $E$ ) of the right common iliac artery (E). Through the right femoral artery and then the right iliac artery with the implanted stent, the delivery catheter system for TAVI was introduced (F), and the valve was implanted at the aortic position.

use of catheter-based lithoplasty by shockwave technology has been recently described (9). A fully percutaneous minimally invasive approach is preferred; however, when a calcified plaque is present in a puncture place of the femoral artery, then it is appropriate to prepare the femoral access with the help of a vascular surgeon. Another important aspect of the procedure is timing, which depends on the patient's symptoms. A staged approach after endothelization of the stent is preferred because of its stable fixation and lower risk of dislocation or impairment during manipulation with a valve delivery system. Nevertheless, in case of severe symptomatic aortic stenosis, it is possible to cautiously deliver a new valve through a recently implanted peripheral vascular stent.

\section{Conclusion}

We suggest recommendations for the strategy of minimally invasive percutaneous transfemoral TAVI combined with PTA for previously contraindicated patients with a poor peripheral vascular access or an attempt to avoid alternative TAVI approaches.

\section{References}

1. Baumgartner H, Falk V, Bax JJ et al. ESC/EACTS guidelines for the management of valvular heart disease. Eur Heart J 2017; 38: 2739-2791.

2. Mack MJ, Leon MB, Smith CR et al. 5-year outcomes of transcatheter aortic valve replacement or surgical aortic valve replacement for high 


\section{$537-540$}

surgical risk patients with aortic stenosis (PARTNER 1): a randomised controlled trial. Lancet 2015; 385: 2477-2484.

3. Reardon MJ, Van Mieghem NM, Popma JJ et al. Surgical or transcatheter aortic-valve replacement in intermediate-risk patients. $\mathrm{N}$ Engl J Med 2017; 376: 1321-1331.

4. Elmaraezy A, Ismail A, Abushouk AI et al. Efficacy and safety of transcatheter aortic valve replacement in aortic stenosis patients at low to moderate surgical risk: a comprehensive meta-analysis. BMC Cardiovasc Disord 2017; 17: 234.

5. Popma JJ, Deeb GM, Yakubov SJ et al. Transcatheter aortic-valve replacement with a self-expanding valve in low-risk patients. N Engl J Med 2019; 380: 1706-1715.
6. Mack MJ, Leon MB, Thourani VH et al. Transcatheter aortic-valve replacement with a balloon-expandable valve in low-risk patients. N Engl J Med 2019; 380: 1695-1705.

7. Biasco L, Ferrari E, Pedrazzini G et al. Access sites for TAVI: patient selection criteria, technical aspects, and outcomes. Front Cardiovasc Med 2018; 5: 88.

8. Chandrasekhar J, Hibbert B, Ruel M et al. Transfemoral vs nontransfemoral access for transcatheter aortic valve implantation: a systematic review and meta-analysis. Can J Cardiol 2015; 31: 1427-1438.

9. Gorla R, Cannone GS, Bedogni $\mathbf{F}$ et al. Transfemoral aortic valve implantation following lithoplasty of iliac artery in a patient with poor vascular access. Catheter Cardiovasc Interv 2019; 9: 140.

Received December 30, 2019. Accepted January 15, 2020. 